\title{
Prediction of Lagrangian dispersion of fluid particles in isotropic turbulent flows using large-eddy simulation method
}

Received: 26 November 2016 / Revised: 9 April 2017 / Published online: 3 June 2017

(C) Springer-Verlag Wien 2017

\begin{abstract}
The single-, two- and multi-particle dispersions in isotropic turbulent flows are investigated using the direct numerical simulation (DNS), filtered DNS (FDNS) and large-eddy simulation (LES) with a spectral eddy viscosity subgrid scale (SGS) model. The contributions of filtering operation and SGS model to the dispersions are separately studied by comparing the statistics obtained from the three methods. The missing SGS motions in LES can be observed to significantly hinder two-particle and four-particle dispersions if the initial separations are less than or comparable to the filter width. A theoretical analysis of the non-monotonic behavior at short time of the one-time, two-point Lagrangian velocity correlation functions with large initial separations based on the Taylor expansion and the Kolmogorov similarity theory is derived, and the Reynolds number effect on the performance of the spectral eddy viscosity SGS model is also investigated. The results show that the SGS model used performs better with increasing Reynolds numbers. It is concluded that the particle SGS model is needed to be developed to correctly capture the Lagrangian two- and multi-point dispersion statistics of fluid particles.
\end{abstract}

\section{Introduction}

Particle-laden turbulent flows exist widely in many environmental applications and industrial processes, such as the pollutant dispersion in the atmosphere, fluidization in process engineering, and aerosol deposition in spray medication. Efficient dispersion of fluid or inertial particles is one of the key characteristics of turbulent flows [1]. In the last few years, the air pollution, especially the PM2.5 pollution has become a severe health and economic problem in developing countries [2,3]. Understanding turbulent dispersion of fine aerosol particles is of great importance for air pollution prevention and control [4].

For particles with finite inertia suspended in flows, the Eulerian-Lagrangian tracking method [5-8] or momentum methods [9] have been systematically developed. For aerosol particles with very fine diameters, the characteristic relaxation timescale tends to vanish; thus, the dynamics of such particles could be considered as passive scalars like fluid particles [10-12]. Turbulent mixing of the passive scalar can usually be investigated from the Eulerian point of view $[13,14]$. However, the tracking of fluid particles is conceptually simple and in immediate connection with the physics of turbulent dispersion and mixing [15-18]. The time integration of the Lagrangian velocity correlation function along the trajectories of fluid particles was seminally introduced by Taylor to relate it to the single-particle or absolute dispersion [15]. The absolute dispersion is defined as the mean-squared displacement of particles from their initial positions. The single-particle dispersion determines

Z. Zhou · J. Chen · G. Jin $(\bowtie)$

LNM, Institute of Mechanics, Chinese Academy of Sciences, Beijing 100190, China

E-mail: gdjin@lnm.imech.ac.cn

Z. Zhou · J. Chen · G. Jin

School of Engineering Science, University of Chinese Academy of Sciences, Beijing 100049, China 
the average rate of growth of the clouds consisting of pollutant particles from their initial positions and the mean concentration field. Theoretically, the single-particle dispersion behaves as ballistic regime at short time and as diffusive regime at long time. Sukhatme [19] studied the Lagrangian velocity correlation function and absolute dispersion in the midlatitude troposphere using wind data from the European Centre for Medium-Range Weather Forecasts (ECMWF). There simultaneously exist superdiffusive and subdiffusive anomalous scalings in the zonal and meridional directions, respectively. The relative dispersion is defined as the mean-squared separation of pairs of particles. Many researchers try to investigate the relative dispersion of particle pairs in isotropic turbulent flows [20,21], in turbulent-like flow fields constructed using the kinematic simulation method [22,23], or experimentally [24]. It is found that the scaling laws of relative dispersion in isotropic turbulent flows depend on the initial separation of particle pairs [25]. In fact, the turbulent relative dispersion is related to the local concentration fluctuations in a chemical mixer, which is very important for the rate of chemical reactions [26,27]. The geometrical properties, such as the evolution of size and shape, are the critical problems for the investigation of a cloud of particles [28-30], which can be studied by tracking the motions of multi-particle clusters, especially the tetrahedrons with four particles [31-34]. Pumir et al. [28] introduced a statistical description of the geometry of the Lagrangian multi-point clusters and predicted a self-similar distribution of shapes. Bianchi et al. [35] studied the evolution of turbulent puffs, each consisting of 2000 particle tracers. Their results show a strong dependence on the fluctuations of the instantaneous wind at the moment of the emission. Yang [36] had systematically studied the temporal evolution of the Lagrangian structures such as the material surface can be tracked as the iso-surface of the Lagrangian scalar field.

The numerical simulation of turbulent dispersion is one of the important methods to predict the efficiency of turbulent mixing. In recent years, the large-eddy simulation (LES) method has become a powerful tool for turbulence prediction [14,37-40]. In LES, the large-scale motions are directly resolved by solving the filtered Navier-Stokes equations with an SGS model for the purpose of closure and to represent the effects of SGS motions on the large-scale ones. Usually, LES can give a reasonable prediction of the turbulent energy spectrum since the construction of an SGS model is based on the energy balance equation and the hypothesis of a $k^{-5 / 3}$ energy spectrum up to the cutoff wavenumber [41,42]. However, LES may not accurately predict the particle dispersion in turbulence due to the lack of velocity fluctuations at small scales. The effects of missing smallscale motions on the dispersion of particles represent a long-standing challenge in LES. There are many studies focusing on the assessment of SGS turbulence effects on the particle motion. The effects of filtering on different statistics of particle motion in isotropic turbulent flows were studied by applying the sharp spectral filter [43] and top filter [44] to the direct numerical simulation (DNS) velocity field. Armenio et al. [45] investigated the effects of small scales of turbulence and SGS model on the motion of tracer particles by releasing particles in a turbulent channel flow and following their motions in time. Compared with inertial particles, the tracer particles are more sensitive to the subgrid scale velocity fluctuations. Fede and Simonin [46] investigated the effects of the subgrid fluid turbulence on the motion of non-settling colliding particles suspended in steady homogeneous isotropic turbulent flows and characterized the statistical properties of the subgrid fluid turbulence experienced by inertial particles. They found that accumulation and collision phenomena for inertial particles are significantly influenced by the subgrid fluid velocity fluctuations. Marchioli et al. [47] addressed some open issues related with the modeling of inertial particle dispersion in LES of turbulent wall-bounded flows and discussed the necessity of using closure models for the equations of particle motion when using LES. The two approaches, DNS and LES of particle-laden turbulent channel flow, in which the particles experience a drag force, were performed by Kuerten and Vreman [48], and the effects of turbophoresis by LES were discussed. Besides, different models such as stochastic model [49], approximate deconvolution method (ADM) [50] and Lagrangian filtered mass density function (LFMDF) model [51] were applied to model the effects of the missing SGS fluid motions in the LES of various particle-laden turbulent flows.

For inertial particles, inertia causes an extra time filtering effect on the fluid velocity experienced by the particle depending on the particle relaxation time. The inertia time filtering effect coupled with the spatial filtering effect makes it difficult to investigate the effects of the small-scale turbulence on the particle motion. Tracking of fluid particles would remove the important contribution from the overall filtering effects felt by the particles. Thus, the statistics of fluid particle dispersion in the present work are determined solely by spatial filtering of the flow field. Yang et al. [52] had investigated the contributions of the spectral eddy viscosity-based SGS model to the Lagrangian dispersion of a single-, two- and four-fluid particle in isotropic turbulence. Their results show that LES can give an accurate prediction of the long-time turbulent dispersion coefficient, while LES tends to underestimate significantly the rate of relative dispersion of particle pairs and multiple-particles if initial separation distances are less than the filter width. The errors in LES come from several sources: (i) the filtering operation on the Navier-Stokes equations, (ii) the SGS model errors, (iii) the interpolation 
error of fluid velocity into the positions of fluid particles, and (iv) the numerical discretization errors of the fluid and particle equations of motions [53-55]. Usually, the effects of these errors are mixed and lead to the errors in the prediction of Lagrangian dispersion. Marchioli et al. [56] have used the fractal interpolation and approximate deconvolution techniques to reduce the effects of the filtering error on near-wall accumulation of inertial particles in turbulent gas flow in a flat channel. Their results show that reconstructing the correct energy spectrum is not enough to represent the effects of SGS motions on particle accumulation, and additional information on the flow field at the subgrid level is needed.

The objective of this work is to separate and further clarify the source of errors in LES of Lagrangian dispersion of fluid particles with focus on the first two errors. We shall use the highly accurate pseudospectral method in the Navier-Stokes equations and a high-order Lagrangian interpolation method for the fluid velocity experienced by fluid particles to neglect the last two. For this purpose, we shall compare the results about Lagrangian dispersion from DNS, filtered DNS (FDNS) and LES with a spectral eddy viscosity model. In the DNS, turbulent flows at the Taylor-based Reynolds number of 205.5 are obtained, much higher than in the previous study [52]. The FDNS is a post-process of the DNS data by filtering out the small-scale motion and can be regarded as an ideal LES without any model error. The results about Lagrangian dispersion of fluid particles consist of single-, two- and four-particle statistics. The relative dispersion and velocity correlations for particle pairs are significantly affected by small-scale fluid motion, which can be applied to evaluate the prediction of particle dispersion by FDNS and LES. The four-particle dispersion describes the evolution of size and shape of tetrahedrons and provides more information on the turbulent transport process than single-particle and two-particle dispersion. The new contributions include a thorough analysis of the non-monotonic behavior of the one-time, two-point Lagrangian velocity correlation functions with large initial separations at short time based on the Taylor expansion of the variance of particle separations in short time and the Kolmogorov similarity theory, and the Reynolds number effect on the performance of the SGS model using the relative error of one-time, two-point Lagrangian correlation functions obtained from FDNS and LES.

This article is organized as follows. In Sect. 2, we present the numerical simulation methods including DNS, FDNS, and LES. The flow field statistics are given in Sect. 3. In Sect. 4, we present the numerical results and discuss the contributions of SGS motions to single-particle, particle-pair and four-particle dispersions in turbulence. The non-monotonic variation of the relative dispersion with a large initial separation at short time is theoretically analyzed based on Taylor series expansion and Kolmogorov similarity hypothesis. In Sect. 5, we shall give the conclusions and possible directions for future work.

\section{Methods}

\subsection{Direct numerical simulation}

The Navier-Stokes equations for isotropic turbulent flows are

$$
\begin{aligned}
\frac{\partial \mathbf{u}}{\partial t} & =\mathbf{u} \times \omega-\nabla\left(\frac{p}{\rho}+\frac{1}{2} \mathbf{u}^{2}\right)+v \nabla^{2} \mathbf{u}+\mathbf{f}(\mathbf{x}, t), \\
\nabla \cdot \mathbf{u} & =0
\end{aligned}
$$

where $\mathbf{u}$ denotes the velocity field, $\omega=\nabla \times \mathbf{u}$ is the vorticity, $p$ is the pressure, $\rho$ is the fluid density, and $v$ is the fluid kinematic viscosity. $\mathbf{f}(\mathbf{x}, \mathbf{t})$ is the large-scale random force used to drive and maintain the turbulent flow.

The DNS of isotropic turbulent flows is performed using a standard pseudo-spectral method in a periodic box of side $L=2 \pi$. In Fourier space, Eqs. (1) and (2) can be represented as $\left(k \leq k_{\max }\right)$

$$
\left(\frac{\partial}{\partial t}+v k^{2}\right) \hat{\mathbf{u}}(\mathbf{k}, t)=\mathbf{P}(\mathbf{k}) \mathcal{F}(\mathbf{u} \times \omega)+\hat{\mathbf{f}}(\mathbf{k}, t)
$$

where $\hat{\mathbf{u}}(\mathbf{k}, t)$ is the Fourier coefficient or the fluid velocity in Fourier space, $\mathcal{F}$ denotes a Fourier transformation, the projection tensor $\mathbf{P}(\mathbf{k})=\delta_{i j}-k_{i} k_{j} / k^{2}(i, j=1,2,3)$ projects $\mathcal{F}(\mathbf{u} \times \boldsymbol{\omega})$ onto the plane normal to $\mathbf{k}$ and eliminates the pressure term in Eq. (1) with the aid of the continuity equation (2), and the maximum wavenumber $k_{\max }$ is about $N / 3$, where $N$ is the number of grid points along each of the three axes. The wavenumber components in Fourier space are $k_{j}=n_{j}(2 \pi / L)$ where $n_{j}=-N / 2, \ldots,-1,0,1, \ldots, N / 2-1$ for $j=1,2,3$. Aliasing errors are removed using the two-thirds truncation method. The spatial resolution is 
monitored by the value of $k_{\max } \eta$, where $\eta$ is the Kolmogorov length scale. The value of $k_{\max } \eta$ should be larger than 1.0 for the Kolmogorov scale of the flow to be well resolved, which is always larger than 1.3 in this work. The Fourier coefficients of the flow velocity are advanced in time using a second-order Adams-Bashforth method for the nonlinear term and an exact integration for the linear viscous term. The time step is chosen to ensure that the CFL number is 0.5 or less for numerical stability and accuracy.

\subsection{Filtered direct numerical simulation}

In order to investigate the effects of small-scale velocity fluctuations on Lagrangian dispersion, we shall filter out the small-scale motions from the full range scale velocity obtained from the above DNS. The filtered DNS (FDNS) velocity field is obtained from the DNS velocity field by truncating the Fourier coefficients larger than the cutoff wavenumber $k_{c}$ with the sharp spectral filter,

$$
\tilde{\boldsymbol{u}}(\mathbf{x}, t)=\sum_{|\mathbf{k}|=k_{0}}^{k_{c}} \hat{\mathbf{u}}(\mathbf{k}, t) e^{i \mathbf{k} \cdot \mathbf{x}},
$$

where $\tilde{\boldsymbol{u}}(\mathbf{x}, t)$ is the filtered velocity in physical space, $k_{0}=1$ is the lowest wavenumber in the DNS. FDNS can be regarded as an ideal LES to study the effects of subgrid scale (SGS) eddies on the statistics of fluid particle motions since FDNS does not contain any eddy viscosity SGS model errors.

\subsection{Large-eddy simulation}

The LES of isotropic turbulent flows is performed with a much coarser grid resolution using the same pseudospectral method and large-scale forcing scheme as the above DNS. The governing equation for LES is given by $\left(|\mathbf{k}| \leq k_{c}\right.$, where $k_{c}$ is the cutoff wavenumber in LES)

$$
\left(\frac{\partial}{\partial t}+\left[v+v_{e}\left(k \mid k_{c}\right)\right] k^{2}\right) \hat{\overline{\mathbf{u}}}(\mathbf{k}, t)=\mathbf{P}(\mathbf{k}) \mathcal{F}(\overline{\mathbf{u}} \times \overline{\boldsymbol{\omega}})+\hat{\mathbf{f}}(\mathbf{k}, t)
$$

where $\overline{\mathbf{u}}$ and $\bar{\omega}$ are the resolved velocity and vorticity in physical space, respectively. A spectral eddy viscosity SGS model is used [41,42],

$$
\begin{aligned}
& v_{e}\left(k \mid k_{c}\right)=v_{e}^{+}\left(k \mid k_{c}\right) \sqrt{E\left(k_{c}\right) / k_{c}}, \\
& v_{e}^{+}\left(k \mid k_{c}\right)=C_{k}^{-3 / 2}\left[0.441+15.2 \exp \left(-3.03 k_{c} / k\right)\right] .
\end{aligned}
$$

The spectral viscosity $v_{e}\left(k \mid k_{c}\right)$ depends on the wavenumber $k$, the maximum wavenumber $k_{c}$, and $E\left(k_{c}\right)$, the value of the energy spectrum function at $k_{c} . C_{k}=2.0$ is taken in this paper. The quantity $E\left(k_{c}\right)$ is calculated from the LES flow field at each time step. The above SGS model is constructed based on the turbulent energy budget equations. The statistical two-point closure theory, that is, the eddy-damped quasi-normal Markovian (EDQNM) approximation is used to construct the above SGS model. The hypothesis of a $k^{-5 / 3}$ energy spectrum up to the cutoff wavenumber $k_{c}$ was used to parameterize the SGS model. This hypothesis is believed to be better fulfilled in turbulent flows with increasing Reynolds number. One can expect that the energy spectrum obtained from the LES with the above SGS model approaches the energy spectrum obtained from the FDNS with increasing Reynolds number. One question is whether or not such a model could predict Lagrangian statistics as the FDNS does in turbulent flows with increasing Reynolds number.

\section{Eulerian statistics of the isotropic turbulent flows}

The Eulerian statistics of flow fields from the DNS, FDNS and LES are listed in Table 1. In the isotropic turbulent flows with zero mean velocity, Taylor-based Reynolds number $R e_{\lambda}=u^{\prime} \lambda / v$, where $u^{\prime}=\sqrt{\left\langle u_{i} u_{i}\right\rangle / 3}$ is the root mean square (rms) of turbulent fluctuating velocity, $\lambda=\left(15 v u^{\prime 2} / \varepsilon\right)^{1 / 2}$ is the Taylor microscale, and $T_{E}=u^{\prime 2} / \varepsilon$ is the eddy turnover time. For the turbulent flow at the same Reynolds number obtained using 
Table 1 Eulerian statistics of flow fields from different cases

\begin{tabular}{lllll}
\hline Case & Case 1 & Case 2 & Case 3 & Case 4 \\
\hline Flow field type & DNS & FDNS & LES & LES \\
Grid number $N$ & $512^{3}$ & - & $128^{3}$ & $64^{3}$ \\
Maximum wavenumber $k_{\max }\left(k_{c}\right)$ & 170 & 42 & 42 & 21 \\
Mesh length $d x$ & 0.012 & - & 0.049 & 0.098 \\
Viscosity $v$ & 0.0010 & 0.0010 & 0.0010 & 0.0010 \\
Taylor-based Reynolds number $R e_{\lambda}$ & 205.51 & - & - & - \\
rms fluctuating velocity $u^{\prime}$ & 0.870 & - & 0.866 & 0.854 \\
Dissipation rate $\varepsilon$ & 0.204 & - & - & - \\
Spatial resolution $k_{\max } \eta$ & 1.432 & - & - & - \\
Kolmogorov length scale $\eta$ & 0.00835 & - & - & - \\
Kolmogorov time scale $\tau_{\eta}$ & 0.06976 & 1.478 & - & - \\
Eulerian integral length scale $L_{E}$ & 1.462 & 5.722 & 3.485 & 3.531 \\
Eddy turnover time $T_{E}$ & 3.701 & & & 3.788 \\
\hline
\end{tabular}

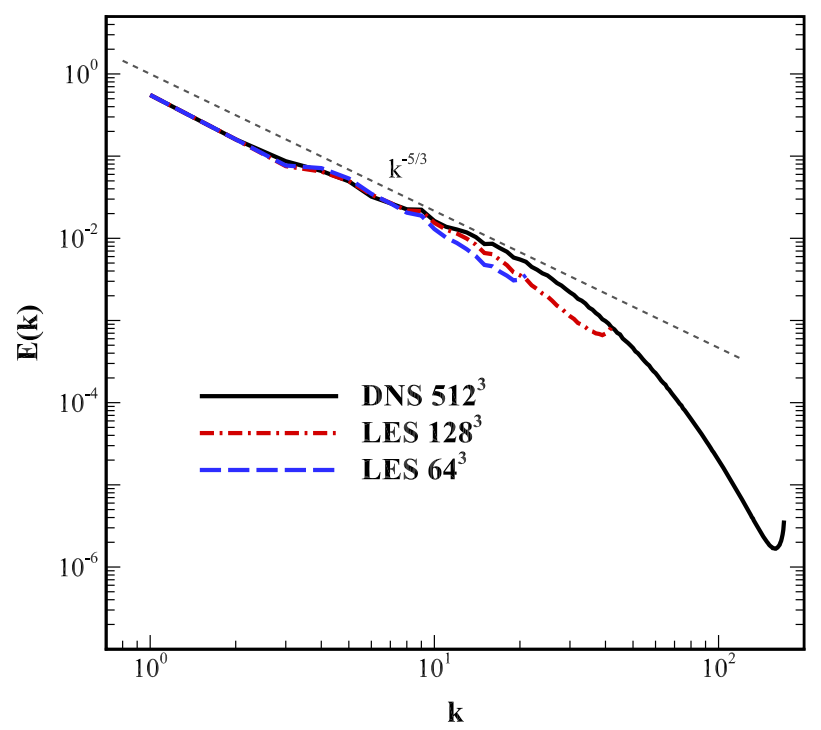

Fig. 1 Energy spectra from DNS (case 1), LES with $128^{3}$-grid resolution (case 3) and LES with $64^{3}$-grid resolution (case 4). The dashed line denotes the classic $k^{-5 / 3}$ turbulent energy spectrum

DNS at a $512^{3}$-grid resolution, two cases of LES at different grid resolutions are performed with the closure of the eddy viscosity model: Case 3 with a $128^{3}$-grid resolution and Case 4 with a $64^{3}$-grid resolution. For the FDNS (Case 2), the cutoff wavenumber is $k_{c}=42$, corresponding to the LES at $128^{3}$-grid resolution (Case 3 ). Figure 1 shows the energy spectra from cases 1, 3, and 4. At large resolved scales, we can observe that the energy spectra from the LES are in good agreement with the one from the DNS. At small resolved scales, the energy spectra from the LES decay faster than the one from the DNS, showing that the eddy viscosity SGS model over-dissipates turbulent energy at high wavenumbers. Moreover, the energy spectra decay faster at coarser resolutions.

\section{Lagrangian dispersion of fluid particles in isotropic turbulent flows}

In this work, we assume that an aerosol particle behaves like a fluid particle, and the trajectory of a fluid particle is calculated by the equation

$$
\frac{\partial \mathbf{X}\left(\mathbf{x}_{0}, t_{0} \mid t\right)}{\partial t}=\mathbf{V}\left(\mathbf{x}_{0}, t_{0} \mid t\right)=\mathbf{u}\left[\mathbf{X}\left(\mathbf{x}_{0}, t_{0} \mid t\right), t\right]
$$

where $\mathbf{X}\left(\mathbf{x}_{0}, t_{0} \mid t\right)$ is the location of the fluid particle at time $t$ which was initially located at $\mathbf{x}_{0}$ at time $t_{0}$, and $\mathbf{V}\left(\mathbf{x}_{0}, t_{0} \mid t\right)$ is the Lagrangian velocity of the fluid particle at time $t$, which equals the Eulerian velocity 


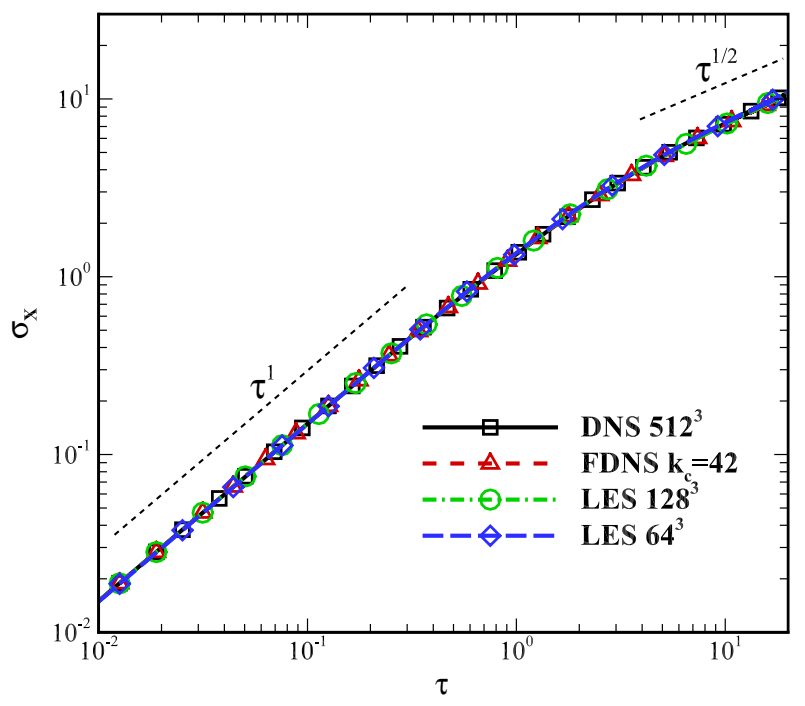

Fig. 2 The standard variance of single-particle displacement. The dashed lines on left bottom and right top have the slopes of 1 and 0.5 , respectively

$u\left[\mathbf{X}\left(\mathbf{x}_{0}, t_{0} \mid t\right), t\right]$ of the flow field at location $\mathbf{X}\left(\mathbf{x}_{0}, t_{0} \mid t\right)$. In order to calculate the velocity of a fluid particle, we make use of a sixth-order Lagrangian interpolation scheme. Then, the trajectory of the fluid particle can be obtained by the explicit fourth-order Adams-Bashforth scheme.

In this work, the particle number is $N_{p}=500,000$ which is initially divided into $N_{p} / 4$ regular tetrahedrons with prescribed side lengths of $1 / 4 \eta, 1 \eta, 8 \eta, 32 \eta$, and $96 \eta$, respectively. The regular tetrahedrons are randomly distributed in the flow field. Each tetrahedron can form six groups of particle pairs with prescribed initial separation. Then, we can track the trajectory of each particle and then calculate the statistics denoting Lagrangian dispersion of single-, two- and four-particles. In addition, we realize several independent samples of Lagrangian statistics starting from different time points during an eddy turnover time to effectively reduce the fluctuations in Lagrangian statistics.

\subsection{Single-particle dispersion}

The variance of single-particle displacement is defined as

$$
\sigma_{X}^{2}(\tau)=\left\langle\sum_{i=1}^{3}\left(X_{i}\left(\mathbf{x}_{0}, t_{0} \mid t_{0}+\tau\right)-x_{0 i}\right)^{2}\right\rangle
$$

where $\sum_{i=1}^{3}\left(X_{i}\left(\mathbf{x}_{0}, t_{0} \mid t_{0}+\tau\right)-x_{0 i}\right)^{2}$ is the square of displacement of the fluid particles at time $t_{0}+\tau$ relative to the initial position $\mathbf{x}_{0}=\left(x_{01}, x_{02}, x_{03}\right), \tau$ is the time interval. According to Taylor [15], the following two asymptotic scaling laws at short and long times are

$$
\begin{cases}\sigma_{X}(t)=\sigma_{V} t, & t \ll T_{L} \\ \sigma_{X}(t)=\sigma_{V}\left(2 T_{L} t\right)^{1 / 2}, & t \gg T_{L}\end{cases}
$$

where $\sigma_{V}^{2}=\left\langle V_{i} V_{i}\right\rangle$ is the variance of the Lagrangian velocity of a fluid particle, and $T_{L}$ is the integral time of the two-time velocity correlation function for a single particle. For a stationary, isotropic turbulent flow, $\sigma_{V}=\sqrt{3} u^{\prime}$. Figure 2 shows the standard variances of the single-particle displacement obtained from the DNS, FDNS, and LES with different grid resolutions. We can observe that the standard variance of single-particle displacement increases with time, and the numerical results validate the theoretical ballistic regime at short time and diffusion regime at long time. Also we observe that the single-particle dispersion is insensitive to the missing small-scale motions, and the spectral eddy viscosity SGS model accurately predicts the particle displacement variance such that the results from DNS, FDNS, and LES collapse together. 


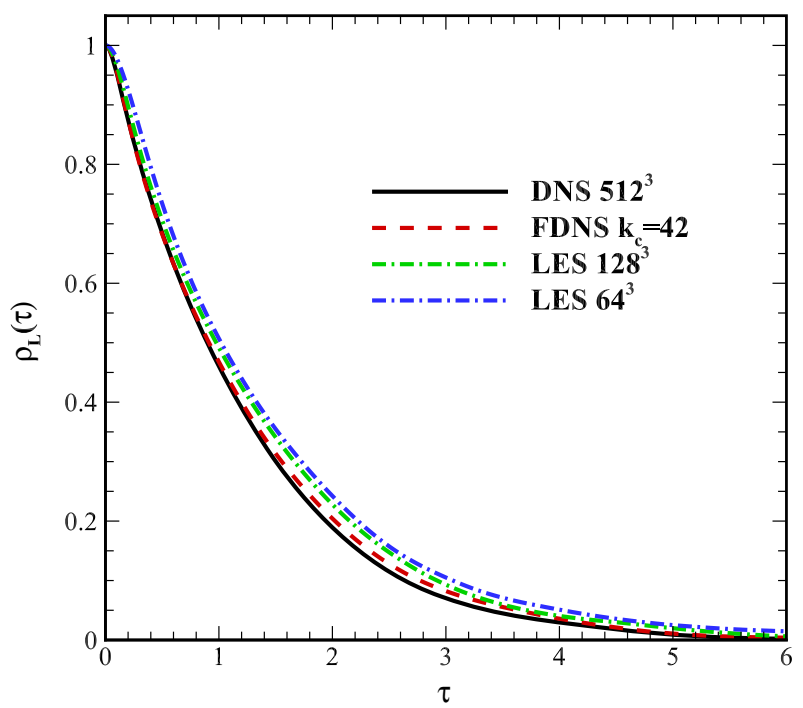

Fig. 3 Lagrangian time correlation functions from DNS at $R e_{\lambda}=205.51$, FDNS, and LES at different resolutions

The Lagrangian velocity correlation function (LVCF) for a single particle is defined as

$$
\rho_{L}(\tau)=\frac{\left\langle V_{i}\left(\mathbf{x}_{0}, t_{0} \mid t_{0}\right) V_{i}\left(\mathbf{x}_{0}, t_{0} \mid t_{0}+\tau\right)\right\rangle}{\sigma_{V}^{2}}
$$

where $V_{i}\left(\mathbf{x}_{0}, t_{0} \mid t_{0}\right) V_{i}\left(\mathbf{x}_{0}, t_{0} \mid t_{0}+\tau\right)$ denotes the dot product of particle velocity vectors at two times, and the angle brackets \langle\rangle denote average over the number of particles. Then, we can calculate the Lagrangian integral time scale by

$$
T_{L}=\int_{0}^{\infty} \rho_{L}(\tau) \mathrm{d} \tau
$$

Figure 3 shows the Lagrangian time correlation functions from the DNS, FDNS, and LES. The correlation function from the FDNS decays slower than those from the DNS, but faster than those from the LES. The former is due to the lack of velocity fluctuations at small scales, whereas the latter is due to both the filtering and the over-dissipation of turbulent energy by the eddy viscosity SGS model. The over-dissipation of the SGS model makes the flow field more coherent and the life of the eddy longer. Besides, the correlation functions from the low-resolution LES $\left(64^{3}\right)$ decay slower than that from the high-resolution LES $\left(128^{3}\right)$ due to the smoother flow field in the former.

\subsection{Two-particle dispersion}

For a particle pair, the relative separation vector $\mathbf{R}$ at one time is defined as

$$
\mathbf{R}\left(\mathbf{r}, t_{0} \mid \tau\right)=\mathbf{X}\left(\mathbf{x}_{0}, t_{0} \mid t_{0}+\tau\right)-\mathbf{X}\left(\mathbf{x}_{0}+\mathbf{r}, t_{0} \mid t_{0}+\tau\right)
$$

where $\mathbf{r}$ is the initial separation vector between the particle pair and $\tau$ is the time interval from the initial time $t_{0}$. The separation distance between the pair particles is calculated from

$$
R\left(\mathbf{r}, t_{0} \mid \tau\right)=\sqrt{\mathbf{R}\left(\mathbf{r}, t_{0} \mid \tau\right) \cdot \mathbf{R}\left(\mathbf{r}, t_{0} \mid \tau\right)}
$$

Then, we calculate the mean and variance of separation distance,

$$
\begin{aligned}
& m_{2}(r, \tau)=\left\langle R\left(\mathbf{r}, t_{0} \mid \tau\right)\right\rangle, \\
& \sigma_{2}^{2}(r, \tau)=\left\langle\left[R\left(\mathbf{r}, t_{0} \mid \tau\right)-\left\langle R\left(\mathbf{r}, t_{0} \mid \tau\right)\right\rangle\right]^{2}\right\rangle=\left\langle R_{i}\left(\mathbf{r}, t_{0} \mid \tau\right) R_{i}\left(\mathbf{r}, t_{0} \mid \tau\right)\right\rangle-m_{2}^{2}(r, \tau),
\end{aligned}
$$




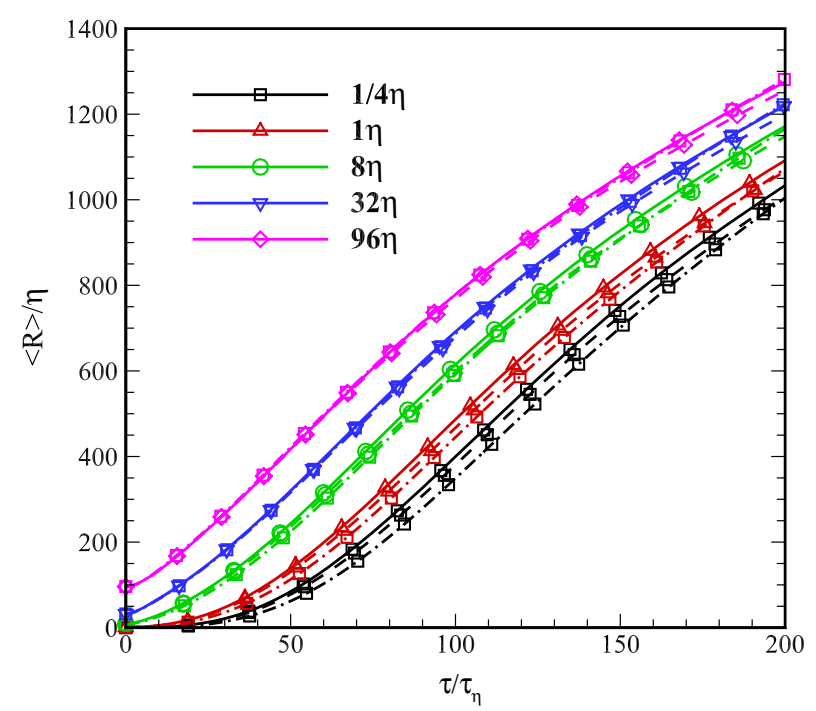

Fig. 4 Time evolution of the mean separations of particle pairs with different initial separation distances: solid lines DNS $512^{3}$, dashed lines FDNS $k_{c}=42$ and dash-dotted lines LES $128^{3}$. Different symbols are corresponding to the different initial separations where $\eta$ denotes the Kolmogorov length scale

where $r$ is the norm of the initial separation vector $\mathbf{r}$. Then, the relative dispersion of a particle pair between initial time $t_{0}$ and time $t_{0}+\tau$ is defined as $\langle\delta \mathbf{R}(r, \tau) \cdot \delta \mathbf{R}(r, \tau)\rangle$, where $\delta \mathbf{R}(r, \tau)=\mathbf{R}\left(\mathbf{r}, t_{0} \mid \tau\right)-\mathbf{r}$ is the separation increment from the initial separation $\mathbf{r}$. There are two regimes for the relative dispersion, which are the Batchelor regime and Richardson regime at very short and long times, respectively,

$$
\langle\delta \mathbf{R}(r, \tau) \cdot \delta \mathbf{R}(r, \tau)\rangle=\left\{\begin{array}{ll}
\frac{11}{3} C_{0} r^{2}\left(\frac{\tau}{t_{a}}\right)^{2}, & \tau \ll t_{a} \equiv\left(\frac{r^{2}}{\varepsilon}\right)^{1 / 3} \\
\mathrm{~g}_{\mathrm{R}} \varepsilon \tau^{3}, & t_{a} \ll \tau \ll T_{E}
\end{array},\right.
$$

where $g_{R}$ is the Richardson constant.

The one-time two-point Lagrangian velocity correlation function of particle pairs with initial separations $\mathbf{r}$ at $t_{0}$ is defined as

$$
\rho_{r}(r, \tau)=\left\langle V_{i}\left(\mathbf{x}_{0}, t_{0} \mid t_{0}+\tau\right) V_{i}\left(\mathbf{x}_{0}+\mathbf{r}, t_{0} \mid t_{0}+\tau\right)\right\rangle / \sigma_{V}^{2}
$$

where $\mathbf{x}_{0}$ is the initial position of the reference particle in a particle pair, $r$ is the magnitude of $\mathbf{r}$, and $\tau$ is the time lag.

The mean and variance of separation distances of particle pairs at five different initial separations $r / \eta=$ 1/4, 1, 8, 32, 96 are shown in Figs. 4 and 5, respectively. In Fig. 4, one can observe that particle pairs with small and moderate separations depart from each other faster in the DNS flow field than in the FDNS flow field, and most slowly separate in the LES flow field, showing that the relative dispersion of the particle pair in the LES flow field is the least efficient one. For the particle pairs with large initial separations such as $r=96 \eta$, the relative dispersion of such particle pairs is mainly controlled by the flow at large scales, so that the mean separation distances in the LES and FDNS are close to the result from DNS. As shown in Fig. 5, all the separation variances obtained from the DNS, FDNS, and LES initially increase with time with a slope equal to 2 on the log-log plot. At long times, the variances increase linearly with time. Between the two limiting regimes, the transient range of smaller initial separations is longer than that of larger initial separations due to the effect of velocity fluctuations at small scales. However, the FDNS and LES underpredict the separation variances compared to the DNS because of the lost small-scale velocity fluctuations. Besides, the separation variances in LES grow more slowly than that in FDNS, because the dissipation of the SGS model further reduces the velocity fluctuations, and the flow field in LES is more coherent.

Pitton et al. [57] studied the particle-laden turbulent gas flow in a channel and examined the turbulent pair dispersion from a statistical point of view. The role of mean shear and small-scale turbulent velocity fluctuations on dispersion is analyzed by focusing on the mean square separation $\left\langle\mathbf{R}\left(\mathbf{r}, t_{0} \mid \tau\right) \cdot \mathbf{R}\left(\mathbf{r}, t_{0} \mid \tau\right)\right\rangle$, where $\mathbf{R}\left(\mathbf{r}, t_{0} \mid \tau\right)$ denotes the separation vector between the particle pair, as described in Eq. (13). Figure 6 


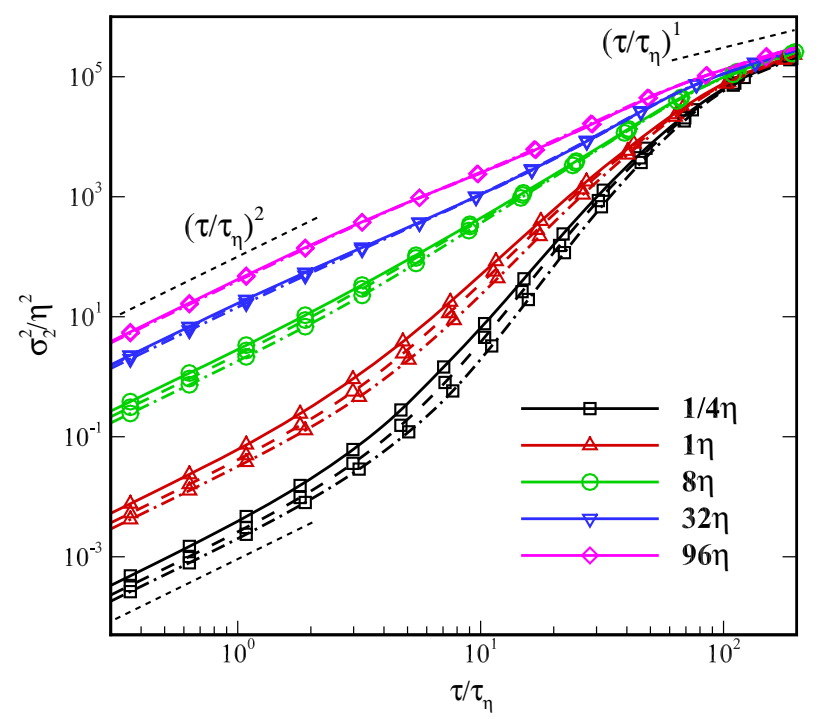

Fig. 5 Time evolution of the variance of separation distances of particle pairs for different initial separation distances: solid lines DNS $512^{3}$, dashed lines FDNS $k_{c}=42$, and dash-dotted lines LES $128^{3}$. The left two dashed straight lines have a slope of 2 , and the right top one has a unity slope

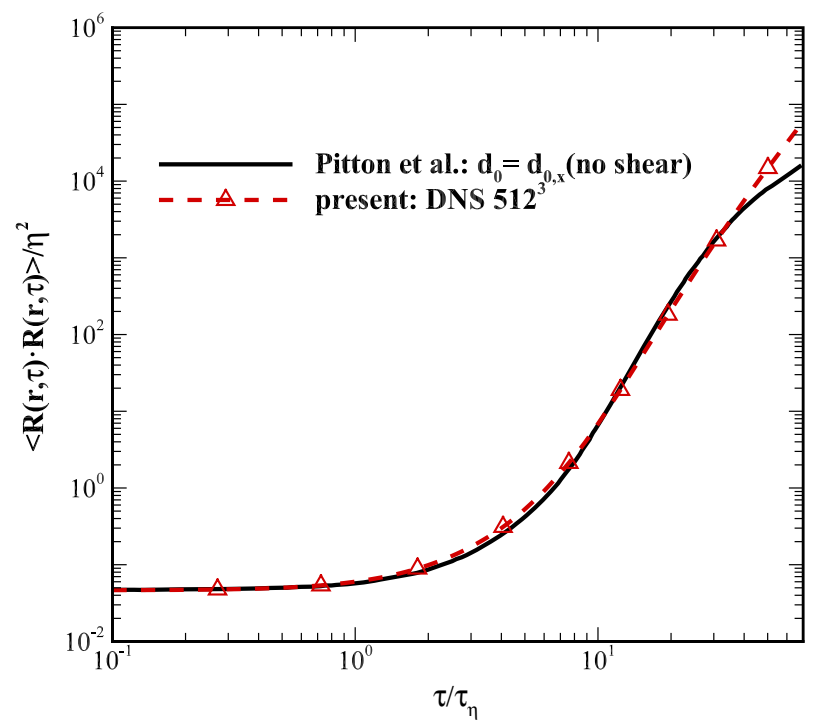

Fig. 6 The comparison of the mean square separation of fluid particle pairs between the present numerical results and the results by Pitton et al. [57]. The solid line denotes the mean square separation results without the shear effects by Pitton et al. [57]. The dashed line with symbols denotes the DNS results of the present work

shows the comparison of the mean square separation of fluid particle pairs between the present numerical results and the results at the center plane of the channel by Pitton et al. [57]. $d_{0, x}$ denotes the initial pair orientation, where $x$-, $y$ - and $z$-directions denote the streamwise, spanwise and wall-normal direction, respectively. The quantities of transverse and longitudinal axis are scaled by the Kolmogorov timescale $\tau_{\eta}$ and Kolmogorov length scale $\eta$ at the center of the channel.

In Fig. 6, the solid line denotes the mean square separation results without the shear effects by Pitton et al. [57]. The results without the shear effects are obtained by tracking particles using only the fluctuating streamwise fluid velocity, which can be calculated by subtracting the mean velocity at the particle location from the fluid particle velocity. The dashed line with symbols denotes the DNS results of the present work. At large times, the results of Pitton et al. without the shear effects differ from the present DNS results, because the particles may move away from the center plane of the channel, and shear plays roles in dispersion. But before 


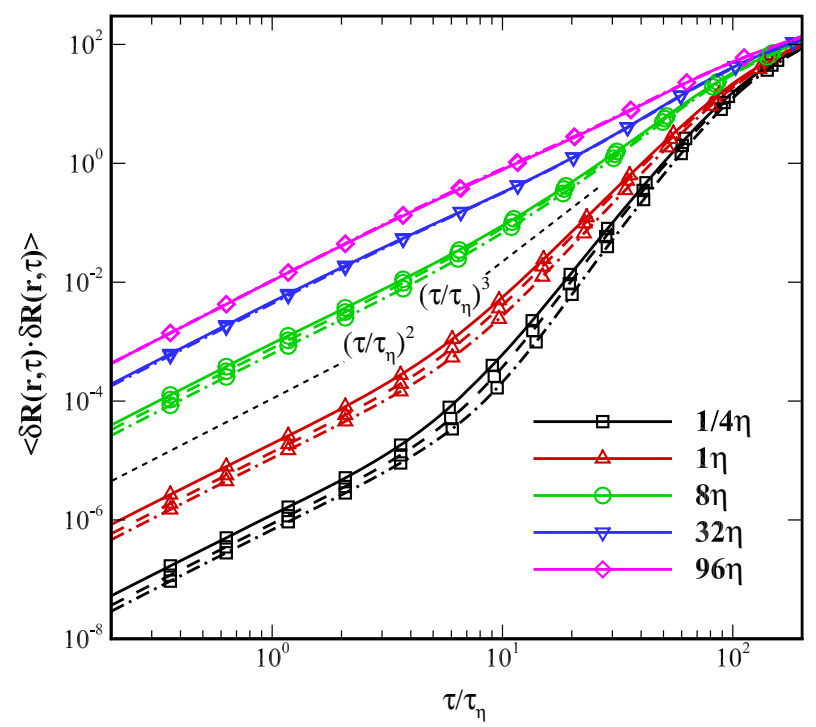

Fig. 7 Time evolution of the relative dispersion of particle pairs for different initial separations: solid lines DNS $512^{3}$, dashed lines FDNS $k_{c}=42$, and dash-dotted lines LES $128^{3}$. The symbols are corresponding to the different initial separations, and $\eta$ denotes the Kolmogorov length scale. The slopes of the left and right dashed lines are equal to 2 and 3, respectively

that, the results of Pitton et al. without the shear effects are consistent with the present DNS results, indicating that the small-scale turbulent fluctuations of the fluid velocity field dominate the particle-pair dispersion near the center of the channel.

Figure 7 plots the temporal evolutions of the relative dispersion of particle pairs from the DNS, LES, and FDNS at different initial separations. At the beginning, the relative dispersion grows with a slope of 2 on the $\log -\log$ plot, which confirms the Batchelor regime. With the increase in time, the slopes of the curves with small initial separations change. At long times, the curves of different initial separations tend to converge. At intermediate ranges, the slopes of the curves with larger initial separations are always smaller than 3. Furthermore, the relative dispersion in FDNS grows with time more slowly than that in DNS but faster than that in LES, just like Fig. 5. It is noted that we cannot observe the Richardson regime for the initial separations in the inertial subrange; this is due to the relatively low Reynolds number in our simulation. The Richardson regime cannot even be observed in laboratory turbulent flows at a much higher Reynolds number of $R e_{\lambda}=815$ [27]. In order to validate our numerical result, we compare the scaled relative dispersion obtained from DNS and the experimental data of Bourgoin et al. [27] by putting our simulation data on their plot in Fig. 8. We can observe that our numerical results agree well with the theoretical and the experimental results.

In Fig. 9, the one-time two-point Lagrangian velocity correlation functions of particle pairs from the DNS, LES, and FDNS at different initial separations are compared. One can observe that the correlation functions from the FDNS decay slower than those from the DNS, but faster than those from the LES. This behavior can be explained by the fact that the flow fields in FDNS and LES are more correlated than that in DNS due to the shortage of small-scale motions. And in the LES flow field, the over-dissipation of energy by the spectral eddy viscosity SGS model leads to a much more coherent flow field and an overprediction of correlation functions $[59,60]$. For large initial separation distances, the differences in the correlation functions obtained from LES, FNDS, and DNS gradually vanish since the large-scale motions of the flow field dominate the dispersion of particle pairs at such separations. We can observe that the correlation functions decrease with time monotonously for particle pairs with small initial separation distances. However, the correlation functions of particle pairs with moderate separations non-monotonically vary with time, for example $r=32 \eta$ and $r=96 \eta$. They slightly increase with time and then decease (Fig. 9b). This phenomenon can be understood due to the fact that part of total particle pairs moves closer to each other after release and becomes more correlated, causing the slight increase in the correlation functions. This fact can be observed from the temporal evolution of the probability density function (PDF) of the separations in Fig. 10, where the vertical solid line denotes the initial separation of $r=96 \eta$. The areas under the curves on the right-hand side of the vertical solid line show that most of the particle pairs will depart from each other, and the peak of the curves moves to larger values of separation with increasing time, while the areas under the curves on the left-hand side of the vertical 


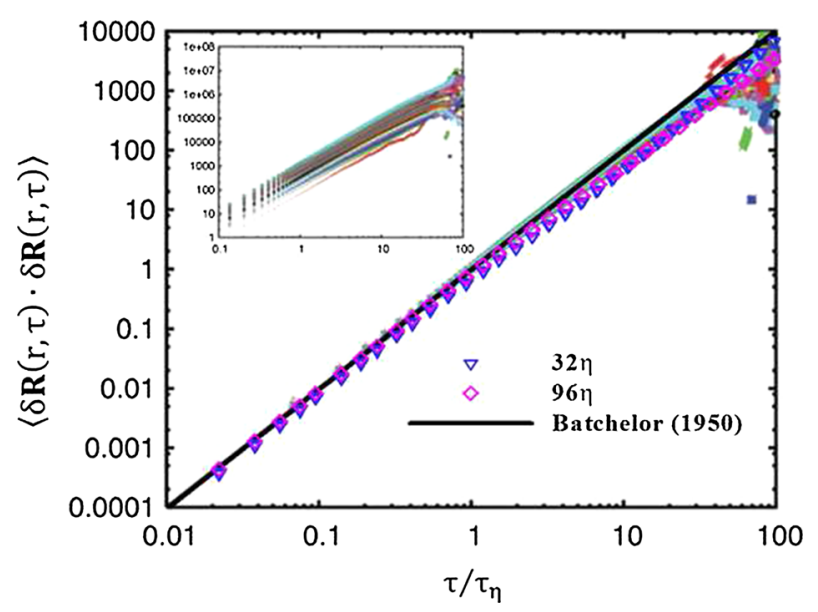

Fig. 8 The comparison of rescaled relative dispersion between the DNS results and experimental results by Bourgoin et al. [27] at turbulent level of $R e_{\lambda}=815$. All the curves are scaled by the constant $11 C_{2}(\varepsilon r)^{2 / 3} / 3$. The solid line denotes the theoretical results of Batchelor [58]. The curves composed of small symbols denote experimental results for 50 different initial separations from $43 \eta$ to $2150 \eta$ or from $0-1$ to $49-50 \mathrm{~mm}$. The symbols denote the DNS results
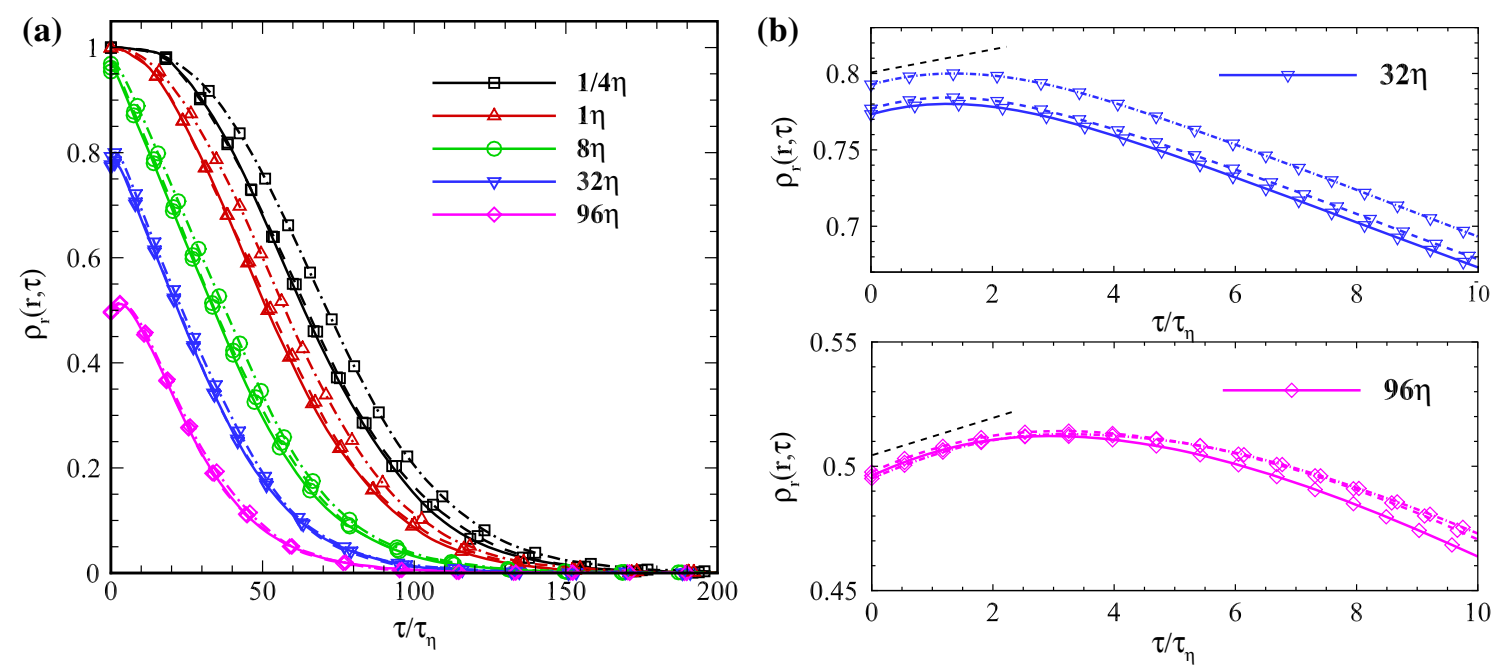

Fig. 9 a The one-time two-point Lagrangian velocity correlation functions of particle pairs for different initial separations: solid lines DNS $512^{3}$, dashed lines FDNS $k_{c}=42$ and dash-dotted lines LES $128^{3}$. The symbols are corresponding to the different initial separations, and $\eta$ denotes the Kolmogorov length scale. b The enlargement of the curves for initial separation $r=32 \eta$ and $r=96 \eta$ to show the non-monotonic variation at short times. The straight dashed lines represent the linear slope at short time, as indicated by Eq. (23)

line represent the probability of the particle pairs which move closer to each other. These approaching particle pairs make one-time two-point Lagrangian velocity correlation functions increase at short time, as shown in Fig. $9 b$.

To further explain the increase in one-time two-point Lagrangian velocity correlation functions at short time in detail, we shall define the separation vector $\mathbf{R}, \mathbf{R}=\mathbf{x}_{p 2}-\mathbf{x}_{p 1}$, the relative velocity $\delta \mathbf{v}=d \mathbf{R} / d t=\mathbf{v}_{p 2}-\mathbf{v}_{p 1}$, and the relative acceleration $\delta \mathbf{a}=d \delta \mathbf{v} / d t=d^{2} \mathbf{R} / d t^{2}$, respectively. Then, the longitudinally relative velocity is $\delta u_{L}=R_{i} \delta u_{i} / R$, and the longitudinally relative acceleration is $\delta a_{L}=R_{i} \delta a_{i} / R$ where $R=\sqrt{R_{i} R_{i}}$ is the separation distance of a particle pair, $\mathbf{v}_{p 1}$ and $\mathbf{v}_{p 2}$ are the velocities of two particles in the pair and $R_{i}$ is the component of vector $\mathbf{R}$. The relation between separation distance and Lagrangian velocity correlation function can be described by 


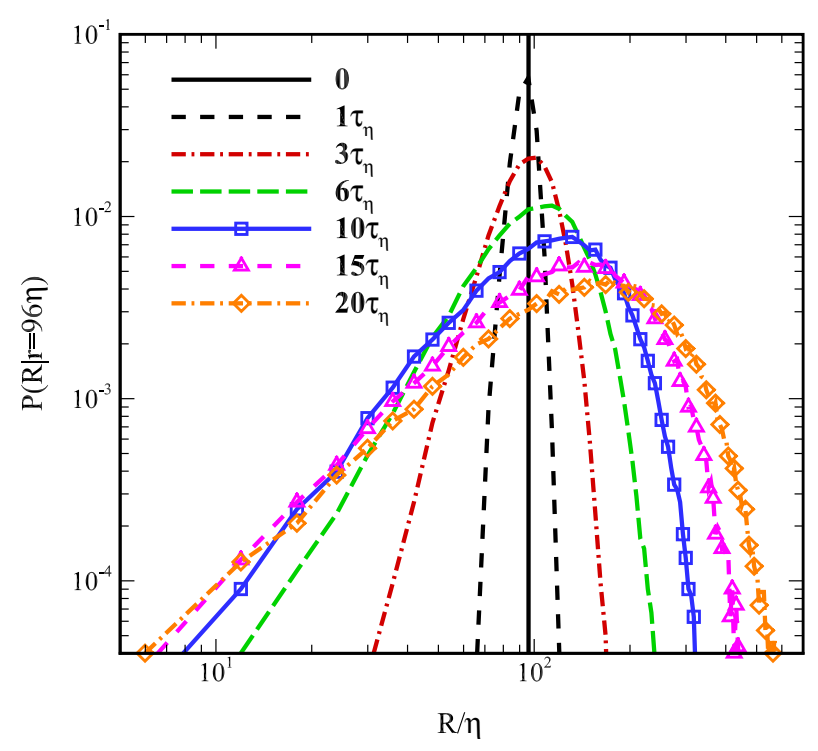

Fig. 10 Temporal evolution of the probability density function (PDF) of particle separations. The vertical solid line represents the initial separations for all particle pairs

$$
\begin{aligned}
& \left\langle\left(\mathbf{v}_{p 2}-\mathbf{v}_{p 1}\right)^{2}\right\rangle=\left\langle\frac{d \mathbf{R}}{d t} \cdot \frac{d \mathbf{R}}{d t}\right\rangle \\
& \frac{\left\langle\mathbf{v}_{p 1}^{2}+\mathbf{v}_{p 2}^{2}-2 \mathbf{v}_{p 1} \cdot \mathbf{v}_{p 2}\right\rangle}{\sigma_{V}^{2}}=\frac{\left\langle\frac{d R}{d t} \frac{d R}{d t}\right\rangle}{\sigma_{V}^{2}} .
\end{aligned}
$$

Here we use the relations $\frac{d R}{d t}=\frac{2 R_{i}}{2 \sqrt{R_{i} R_{i}}} \frac{d R_{i}}{d t}=\frac{R_{i}}{R} \frac{d R_{i}}{d t}$ and $\frac{d R}{d t} \frac{d R}{d t}=\frac{R_{i}}{R} \frac{d R_{i}}{d t} \frac{R_{i}}{R} \frac{d R_{i}}{d t}=\frac{d R_{i}}{d t} \frac{d R_{i}}{d t}=\frac{d \mathbf{R}}{d t} \cdot \frac{d \mathbf{R}}{d t}$.

For isotropic turbulence, $\sigma_{V}^{2}=\mathbf{v}_{p 1}^{2}=\mathbf{v}_{p 2}^{2}$, we can obtain

$$
\rho_{r}=1-\frac{\left\langle\frac{d R}{d t} \frac{d R}{d t}\right\rangle}{2 \sigma_{V}^{2}} .
$$

We take the Taylor expansion of $\left\langle\frac{d R}{d t} \cdot \frac{d R}{d t}\right\rangle$ to the first-order approximation with a very short time $t$ as follows:

$$
\left\langle\frac{d R}{d t} \cdot \frac{d R}{d t}\right\rangle=\left\langle\left.\left(\frac{d R}{d t} \frac{d R}{d t}\right)\right|_{t=0}\right\rangle+\left\langle\left.\left(2 \frac{d R}{d t} \frac{d^{2} R}{d t^{2}}\right)\right|_{t=0}\right\rangle t+\cdots .
$$

We have $\frac{d R}{d t}=\frac{R_{i}}{R} \frac{d R_{i}}{d t}=\frac{R_{i}}{R} \delta v_{i}=\delta v_{L}$, and $\frac{d^{2} R}{d t^{2}}=\frac{d \delta v_{L}}{d t}$. According to the Kolmogorov similarity theory, the following expressions exist for the separations located in the inertial subrange: $\left\langle\left(\delta v_{L}\right)^{2}\right\rangle=C \cdot(\varepsilon R)^{2 / 3}$ and $\left\langle\delta v_{L} \frac{d \delta v_{L}}{d t}\right\rangle=-\frac{6}{5} \varepsilon$. Introducing these relationships into Eq. (22), we obtain the expression

$$
\rho_{r}=1-\frac{C \cdot(\varepsilon r)^{2 / 3}}{6 u^{\prime 2}}+\frac{2 \varepsilon}{5 u^{\prime 2}} t .
$$

Equation (23) demonstrates that the one-time two-point correlation function initially increases slightly for the separations located in the inertial subrange. In Fig. 9b, the predicted slope of the correlation functions is plotted with dashed lines.

In order to investigate the effects of the turbulent Reynolds number on the performance of the spectral SGS model for LES to predict particle-pair dispersion, we calculate the relative error of Lagrangian velocity correlation functions of particle pairs between the LES and FDNS as $\Delta \rho_{r} / \rho_{r \text { FDNS }}=\left(\rho_{r \text { LES }}-\rho_{r \text { FDNS }}\right) / \rho_{r \text { FDNS }}$. The cutoff wavenumber is set to $k_{c}=42$ for the LES and FDNS at different Reynolds numbers. We plot the results at two initial separations $r=\eta$ and $r=4 \eta$ in Fig. 11, from which we can observe that the 

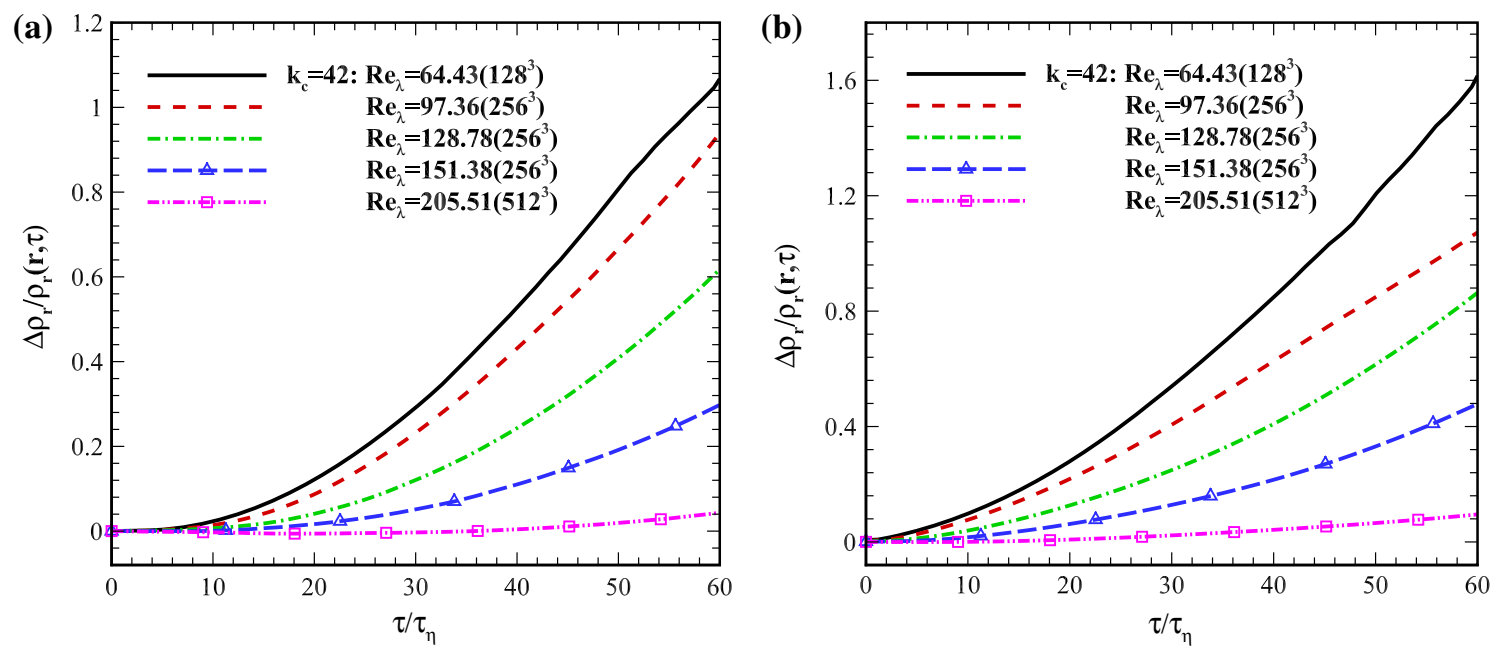

Fig. 11 The relative error of Lagrangian velocity correlation functions of particle pairs between the LES and FDNS with the same cutoff wavenumber $k_{c}=42$ at different Reynolds numbers for different initial separations: $\mathbf{a} r=\eta$ and $\mathbf{b} r=4 \eta$. The numbers in the parentheses () of line legend denote the grid resolutions for the DNS of different Reynolds numbers

relative error decreases with increasing Reynolds numbers. This is expected since the current spectral SGS viscosity model is developed with the aid of the two-point closure theory, and the hypothesis of a $k^{-5 / 3}$ energy spectrum up to the cutoff wavenumber was used, which is more accurate at higher Reynolds numbers.

For further validating the observations about the relative dispersion of particle pairs in the flow fields obtained from FDNS, LES, and DNS, the PDFs of the separation distances at the same time $\tau=20 \tau_{\eta}$ for five initial separations are plotted in Fig. 12. It is observed that the PDF obtained from the LES exhibits a higher peak and a lower tail than the DNS and FDNS, showing a slower dispersion in flow fields obtained on LES than in FDNS and DNS. At small initial separations, the dispersion of particle pairs is dominated by small-scale motions, so that the absence of small-scale motions in the FDNS and the further reduction in small-scale velocity fluctuations by the SGS model in the LES cause the deviations in PDF of separations between the DNS, FDNS, and LES. However at large initial separations, the PDF from the DNS, FDNS, and LES tends to coincide because the large-scale motions dominate the relative dispersion of particle pairs. Furthermore, the higher peak and lower tail can be observed in the LES with coarser grid (LES, $64^{3}$ ) than those in the LES with finer grid (LES, $\left.128^{3}\right)$.

\subsection{Four-particle dispersion}

Multi-particle dispersion could provide more information on turbulent transport processes than single-particle and two-particle dispersion. Here we shall study the time evolution of the size and shape of tetrahedrons each formed by four-fluid particles. Our purpose is to investigate the variations of tetrahedrons with an initial side length $\eta$ in turbulent flows by FDNS and LES, in comparison with the DNS. The statistics are the mean surface area, volume of tetrahedrons, and their renormalized ratios.

A regular tetrahedron is formed by a reference particle at $\mathbf{X}_{0}^{(1)}=\left(x_{0}, y_{0}, z_{0}\right)$ and other three particles,

$$
\left\{\begin{array}{l}
\mathbf{X}_{0}^{(2)}=\left\{x_{0}-\frac{\sqrt{3}}{6} \eta, y_{0}-\frac{1}{2} \eta, z_{0}-\frac{\sqrt{6}}{3} \eta\right\}, \\
\mathbf{X}_{0}^{(3)}=\left\{x_{0}-\frac{\sqrt{3}}{6} \eta, y_{0}+\frac{1}{2} \eta, z_{0}-\frac{\sqrt{6}}{3} \eta\right\}, \\
\mathbf{X}_{0}^{(4)}=\left\{x_{0}+\frac{\sqrt{3}}{3} \eta, y_{0}, z_{0}-\frac{\sqrt{6}}{3} \eta\right\} .
\end{array}\right.
$$



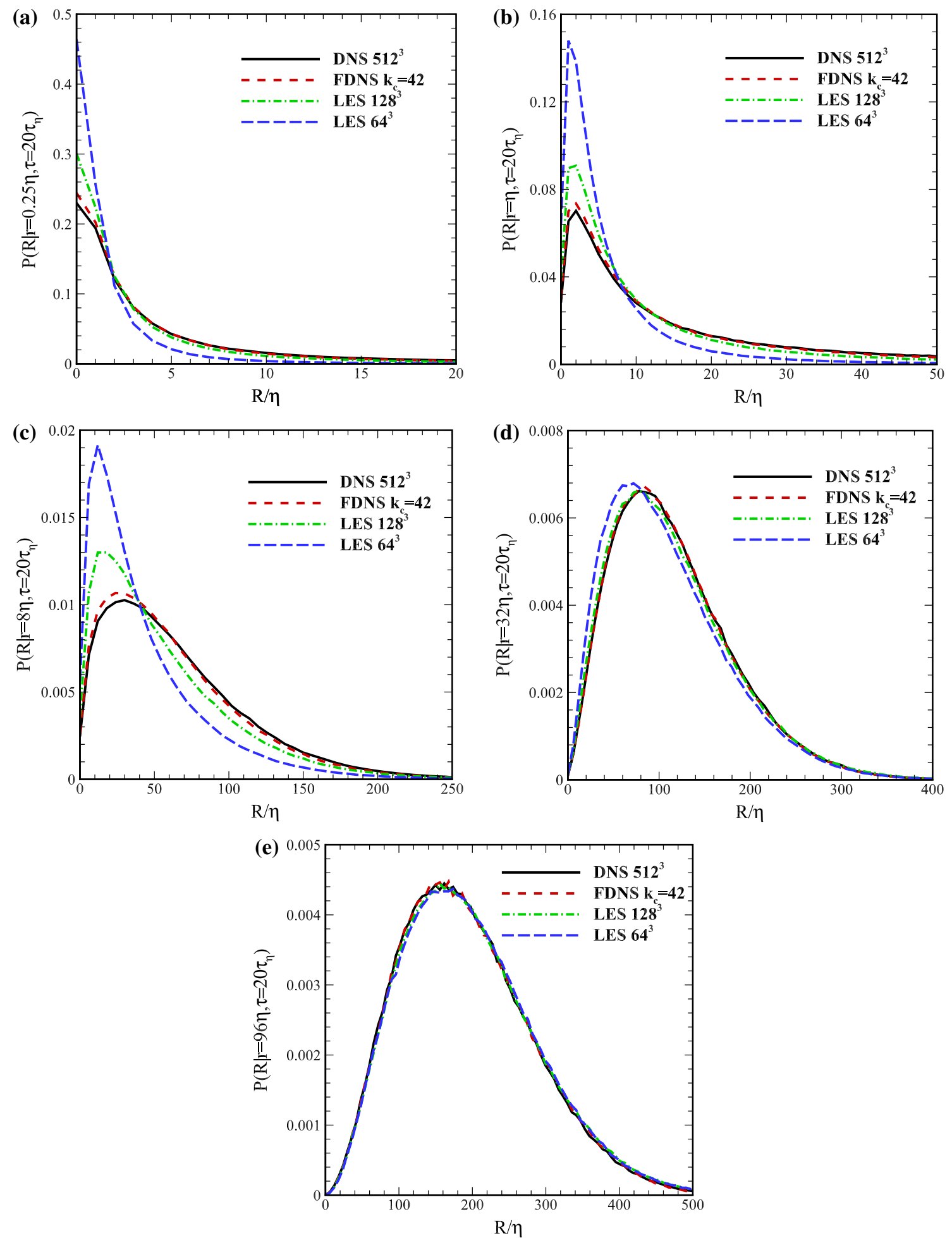

Fig. 12 The PDFs of the separation distances at $\tau=20 \tau_{\eta}$ for the different initial separations: $\mathbf{a} r=0.25 \eta, \mathbf{b} r=\eta$, $\mathbf{c} r=8 \eta$, d $r=32 \eta$, e $r=96 \eta$ 


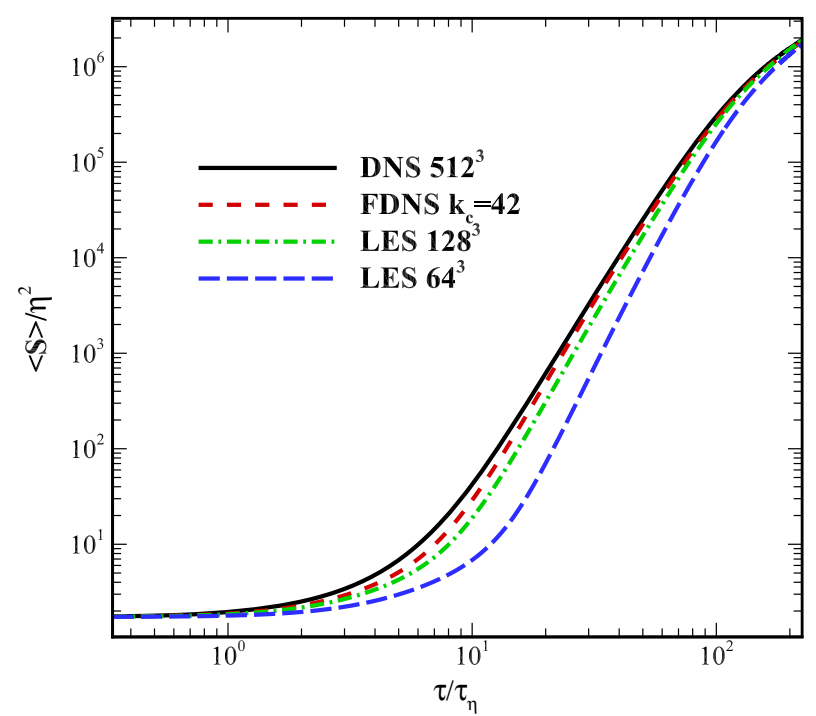

Fig. 13 The mean surface areas of tetrahedrons from the DNS, FDNS, and LES

The area of the triangle formed by the first three particles can be calculated by

$$
\begin{aligned}
S^{(123)}= & \frac{1}{2}\left\{\left[\left(y^{(2)}-y^{(1)}\right)\left(z^{(3)}-z^{(1)}\right)-\left(z^{(2)}-z^{(1)}\right)\left(y^{(3)}-y^{(1)}\right)\right]^{2}\right. \\
& +\left[\left(z^{(2)}-z^{(1)}\right)\left(x^{(3)}-x^{(1)}\right)-\left(x^{(2)}-x^{(1)}\right)\left(z^{(3)}-z^{(1)}\right)\right]^{2} \\
& \left.+\left[\left(x^{(2)}-x^{(1)}\right)\left(y^{(3)}-y^{(1)}\right)-\left(y^{(2)}-y^{(1)}\right)\left(x^{(3)}-x^{(1)}\right)\right]^{2}\right\}^{\frac{1}{2}}
\end{aligned}
$$

where the superscript " 123 " denotes the serial numbers of the particles forming the triangle. The areas of the other three triangles $S^{(124)}, S^{(134)}$ and $S^{(234)}$ can be calculated similarly. The total surface area of one tetrahedron is $S=S^{(123)}+S^{(124)}+S^{(134)}+S^{(234)}$.

The volume of the tetrahedron can be calculated by

$$
V=\frac{1}{6}\left|\begin{array}{lll}
x^{(2)}-x^{(1)} & x^{(3)}-x^{(1)} & x^{(4)}-x^{(1)} \\
y^{(2)}-y^{(1)} & y^{(3)}-y^{(1)} & y^{(4)}-y^{(1)} \\
z^{(2)}-z^{(1)} & z^{(3)}-z^{(1)} & z^{(4)}-z^{(1)}
\end{array}\right| .
$$

In the simulations, the mean surface area $\langle S\rangle$ and volume $\langle V\rangle$ are obtained from 25,000 tetrahedrons with initial side length $\eta$. Figures 13 and 14 show the temporal evolution of the normalized mean surface area $\langle S\rangle / \eta^{2}$ and volume $\langle V\rangle / \eta^{3}$ from the DNS, FDNS, and LES. At the initial stage, there is a little change for the size and shape of the tetrahedrons due to the local incompressible velocity fields, so that the mean surface area and volume exhibit little change for all the simulations. At $t>3 \tau_{\eta}$, the two quantities start to grow because of the two-point dispersion behavior, see Fig. 7. The dispersion increases in the sequence of low-resolution LES $\left(64^{3}\right)$, high-resolution LES $\left(128^{3}\right)$, FDNS, and DNS at the same time. In the FDNS, the small-scale motions are filtered out, which causes the lack of small-scale velocity fluctuations. In the LES, the turbulence is resolved on the coarser grid, and the dissipation by the eddy viscosity SGS model further reduces the velocity fluctuations. These facts lead to the underpredictions of the four-particle dispersion.

The size and shape of a tetrahedron can also be characterized by the renormalized volume ratio $\lambda_{V}$ and surface area ratio $\lambda_{S}$,

$$
\lambda_{V}=V / S^{3 / 2}, \quad \lambda_{S}=S / C^{2},
$$

where $C$ is the perimeter of the tetrahedron. It is known that the regular tetrahedron has the largest ratios. Meanwhile, there are two extreme cases about the ratios: $\lambda_{V}=0$ for the tetrahedron with four-particles coplanar and $\lambda_{S}=0$ for the tetrahedron with four-particles collinear. Figure 15 plots the temporal evolution of the mean ratios $\left\langle\lambda_{V}\right\rangle$ and $\left\langle\lambda_{S}\right\rangle$ of the initially regular tetrahedrons. 


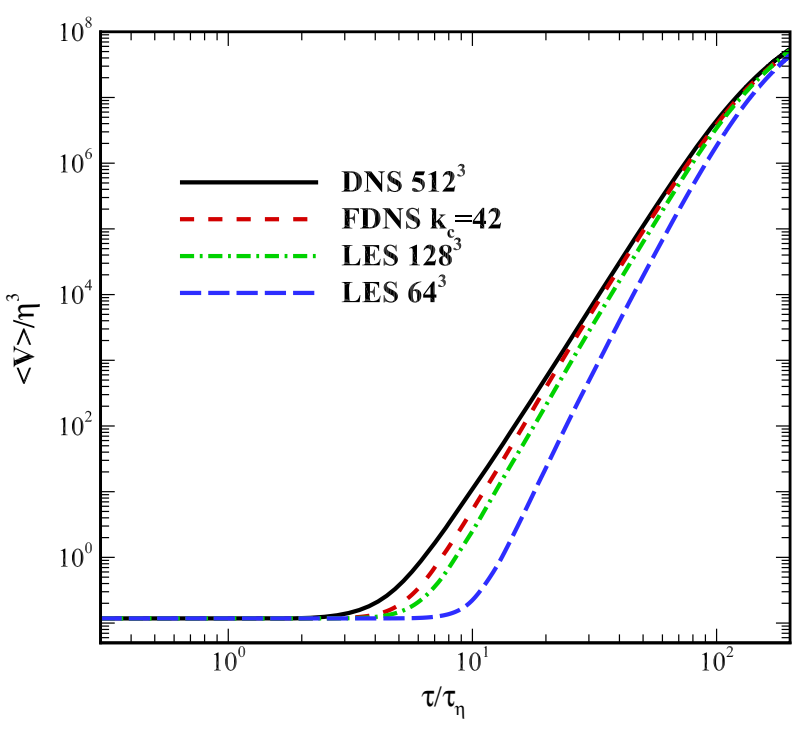

Fig. 14 The mean volumes of tetrahedrons from the DNS, FDNS, and LES

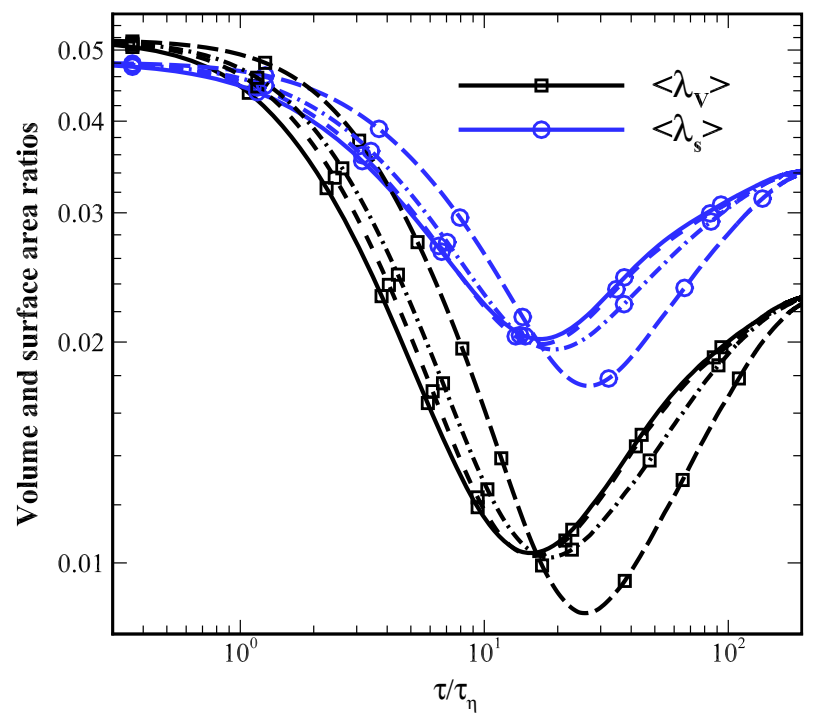

Fig. 15 Temporal evolution of mean renormalized volume and surface area ratios from DNS, FDNS, and LES: solid lines DNS $512^{3}$, dashed lines FDNS $k_{c}=42$, dash-dotted lines LES $128^{3}$, and long-dashed lines LES $64^{3}$

At the initial stage, the volume and surface ratios from the DNS, FDNS, and LES basically coincide with each other and exhibit little changes because the local velocity fields preserve the size and shape of the tetrahedrons. As time increases, the tetrahedrons are dispersed by turbulent stretching, so that the ratios drop to the lowest point. After that, the ratios start to gradually increase. Finally, the results from the DNS, FDNS, and LES tend to converge because most of the side lengths of tetrahedrons correspond to the Eulerian integral length scale so that the shapes of the tetrahedrons are governed by large-scale motions of turbulent flows. During the intermediate stages, the change in the ratios from FDNS is always slower than that from DNS and faster than that from LES. As described in Fig. 12b, the PDF of separation of FDNS exhibits a higher peak than the PDF of the separations from DNS and a highest peak from LES, where a higher peak in PDF means slower dispersion of particle pairs. The distorted tetrahedron in LES is most likely to have edges comparable to subgrid scales. Therefore, the changes in size and shape of a tetrahedron in LES are slower than those in FDNS and DNS. 


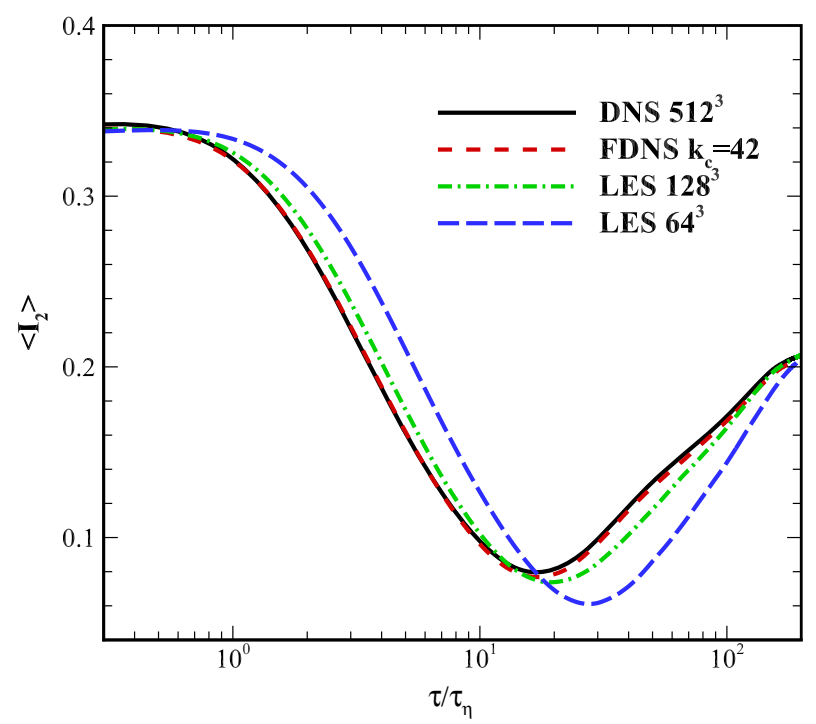

Fig. 16 Temporal evolution of the ratio $\left\langle I_{2}\right\rangle$ from DNS, FDNS, and LES

For a tetrahedron, another indicator for the shape variation is the ratio of the intermediate eigenvalue of the inertia matrix $\mathbf{I}=\rho \rho^{T}$, where

$$
\rho=\left(\begin{array}{ccc}
\rho_{x}^{(1)} & \rho_{x}^{(2)} & \rho_{x}^{(3)} \\
\rho_{y}^{(1)} & \rho_{y}^{(2)} & \rho_{y}^{(3)} \\
\rho_{z}^{(1)} & \rho_{z}^{(2)} & \rho_{z}^{(3)}
\end{array}\right)
$$

with $\rho^{(i)}=\left(\rho_{x}^{(i)}, \rho_{y}^{(i)}, \rho_{z}^{(i)}\right), i=1,2,3$, and $\rho^{(1)}=\left(\mathrm{x}^{(2)}-\mathrm{x}^{(1)}\right) / \sqrt{2}, \rho^{(2)}=\left(2 \mathrm{x}^{(3)}-\mathrm{x}^{(1)}-\mathrm{x}^{(2)}\right) / \sqrt{6}$, $\rho^{(3)}=\left(3 \mathrm{x}^{(4)}-\mathrm{x}^{(1)}-\mathrm{x}^{(2)}-\mathrm{x}^{(3)}\right) / \sqrt{12}[29]$.

The three eigenvalues of the inertia matrix $\mathbf{I}$ are denoted as $g_{1}, g_{2}$, and $g_{3}$ from large value to small one, so the volume $V=\frac{1}{3} \operatorname{det}(\rho)=\frac{1}{3} \sqrt{g_{1} g_{2} g_{3}}$ and the ratio $I_{i}=g_{i} / G^{2}$, where $G=\sqrt{\operatorname{tr}(\mathbf{I})}=\sqrt{g_{1}+g_{2}+g_{3}}$ is the characteristic size of a tetrahedron. For a regular tetrahedron, $I_{1}=I_{2}=I_{3}=\frac{1}{3}$. For the two extreme cases, the four-point coplanar case, $I_{3}=0$, and the four-point collinear case, $I_{2}=I_{3}=0$. Figure 16 shows the temporal evolution of the ratio $\left\langle I_{2}\right\rangle$ from DNS, FDNS, and LES. The change in the ratio $I_{2}$ from FDNS is a little slower than that from DNS and faster than that from LES. And the trends of the curves are similar to the curves of the renormalized mean volume and surface area ratios as shown in Fig. 15.

\section{Conclusions}

In this work, the Lagrangian dispersions of single-, two- and four-particles in isotropic turbulent flows are investigated using direct numerical simulation (DNS), filtered DNS (FDNS) and large-eddy simulation (LES). We aim to separate and study the error sources of the turbulent dispersion obtained from the LES method which has become more and more important in the prediction of pollutant dispersion in the atmosphere and chemical mixing in process engineering. The FDNS can be regarded as an ideal LES without any subgrid scale (SGS) model errors, and the comparison between the statistics obtained from FDNS and DNS can be used to study the contributions of small-scale motions to turbulent dispersion of fluid particles, while the comparison between the statistics obtained from FDNS and LES can be used to study the contributions of SGS model errors to turbulent dispersion of fluid particles. LES usually underpredicts the relative turbulent dispersion and multiparticle dispersion when the initial separations are smaller than or comparable to the width of the filter. This is because the missing small-scale motions in LES have significant effects on the relative dispersion of particle pairs. The Reynolds number-dependent relative error of Lagrangian velocity correlation functions of particle pairs between the LES and FDNS shows that the current spectral SGS model becomes more accurate with increasing Reynolds number. The theoretical analysis based on Taylors expansion of the relative separation 
and the Kolmogorov similarity theory demonstrates the non-monotonic variation of the one-time two-point Lagrangian velocity correlation functions of particle pairs at short time.

Due to the importance of the missing SGS motions on particle relative dispersion, we need to construct corresponding particle SGS models to recover the effects of the missing SGS motions on particle dispersions. Recently, the efforts have been made for both fluid and inertial particles in this direction, including the stochastic model [61,62], deconvolution method [63,64], the hybrid method [65], and the kinematical simulations [66, 67]. We shall develop particle SGS models for LES of Lagrangian dispersion of fluid particles by developing such models based on the space-time correlation models [40,59,68-70] which are necessary for two- or multiparticle dispersion.

Acknowledgements This work was supported by the National Natural Science Foundation of China (11472277, 11572331 and 11232011), Science Challenge Project (TZ2016001), Strategic Priority Research Program, CAS (XDB22040104), Key Research Program of Frontier Sciences, CAS (QYZDJ-SSW-SYS002), and the 973 Program of China (2013CB834100). The anonymous reviewers are much appreciated for the constructive suggestions to improve the manuscript.

\section{References}

1. Dimotakis, P.E.: Turbulent mixing. Annu. Rev. Fluid Mech. 37, 329-356 (2005)

2. Wang, G.Z., Gu, S.J., Chen, J.B., Wu, X.H., Yu, J.: Assessment of health and economic effects by PM2.5 pollution in Beijing: a combined exposure-response and computable general equilibrium analysis. Environ. Technol. 37, 3131-3138 (2016)

3. Kanniah, K.D., Kaskaoutis, D.G., San Lim, H., Latif, M.T., Zaman, N.A.F.K., Liew, J.: Overview of atmospheric aerosol studies in Malaysia: known and unknown. Atmos. Res. 182, 302-318 (2016)

4. Csanady, G.T.: Turbulent diffusion in the environment. D. Reidel Pub. Co., Dordrecht, Boston, Geophysics and astrophysics monographs (1973)

5. Marchioli, C., Soldati, A., Kuerten, J.G.M., Arcen, B., Tanière, A., Goldensoph, G., Squires, K.D., Cargnelutti, M.F., Portela, L.M.: Statistics of particle dispersion in direct numerical simulations of wall-bounded turbulence: results of an international collaborative benchmark test. Int. J. Multiph. Flow 34, 879-893 (2008)

6. Wang, L.P., Ayala, O., Rosa, B., Grabowski, W.W.: Turbulent collision efficiency of heavy particles relevant to cloud droplets. New J. Phys. 10, 075013 (2008)

7. Parishani, H., Ayala, O., Rosa, B., Wang, L.P., Grabowski, W.W.: Effects of gravity on the acceleration and pair statistics of inertial particles in homogeneous isotropic turbulence. Phys. Fluids 27, 033304 (2015)

8. Rosa, B., Parishani, H., Ayala, O., Wang, L.P.: Settling velocity of small inertial particles in homogeneous isotropic turbulence from high-resolution DNS. Int. J. Multiph. Flow 83, 217-231 (2016)

9. Yu, M.Z., Lin, J.Z., Chan, T.L.: A new moment method for solving the coagulation equation for particles in Brownian motion. Aerosol Sci. Technol. 42, 705-713 (2008)

10. Cassiani, M., Stohl, A., Eckhardt, S.: The dispersion characteristics of air pollution from the world's megacities. Atmos. Chem. Phys. 13, 9975-9996 (2013)

11. Leroy-Cancellieri, V., Augustin, P., Filippi, J.B., Mari, C., Fourmentin, M., Bosseur, F., Morandini, F., Delbarre, H.: Evaluation of wildland fire smoke plume dynamics and aerosol load using UV scanning lidar and fire-atmosphere modelling during the Mediterranean Letia 2010 experiment. Nat. Hazards Earth Syst. 14, 509-523 (2014)

12. Cheng, K.C., Park, H.K., Tetteh, A.O., Zheng, D., Ouellette, N.T., Nadeau, K.C., Hildemann, L.M.: Mixing and sink effects of air purifiers on indoor PM2.5 concentrations: a pilot study of eight residential homes in Fresno, California. Aerosol Sci. Technol. 50, 835-845 (2016)

13. Liu, Y., Feng, H., Olsen, M.G., Fox, R.O., Hill, J.C.: Turbulent mixing in a confined rectangular wake. Chem. Eng. Sci. 61, 6946-6962 (2006)

14. Jansen, K.M., Kong, B., Fox, R.O., Hill, J.C., Olsen, M.G.: Large eddy simulation of passive scalar transport in a high Schmidt number turbulent incompressible wake with experimental validation. Chem. Eng. Sci. 137, 862-874 (2015)

15. Taylor, G.I.: Diffusion by continuous movements. Proc. Lond. Math. Soc. 20, 196-212 (1922)

16. Richardson, L.F.: Atmospheric diffusion shown on a distance-neighbour graph. Proc. R. Soc. A 110, $709-737$ (1926)

17. Batchelor, G.K.: Diffusion in a field of homogeneous turbulence. 2. The relative motion of particles. Proc. Camb. Philos. Soc. 48, 345-362 (1952)

18. Toschi, F., Bodenschatz, E.: Lagrangian properties of particles in turbulence. Annu. Rev. Fluid Mech. 41, 375-404 (2009)

19. Sukhatme, J.: Lagrangian velocity correlations and absolute dispersion in the midlatitude troposphere. J. Atmos. Sci. 62, 3831-3836 (2005)

20. Kraichnan, R.H.: Dispersion of particle pairs in homogeneous turbulence. Phys. Fluids 9, 1937-1943 (1966)

21. Thomson, D.J.: A stochastic model for the motion of particle pairs in isotropic high-Reynolds-number turbulence, and its application to the problem of concentration variance. J. Fluid Mech. 210, 113-153 (1990)

22. Fung, J.C.H., Vassilicos, J.C.: Two-particle dispersion in turbulentlike flows. Phys. Rev. E 57, 1677-1690 (1998)

23. Thomson, D.J., Devenish, B.J.: Particle pair separation in kinematic simulations. J. Fluid Mech. 526, 277-302 (2005)

24. Ott, S., Mann, J.: An experimental investigation of the relative diffusion of particle pairs in three-dimensional turbulent flow. J. Fluid Mech. 422, 207-223 (2000)

25. Yeung, P.K.: Direct numerical simulation of two-particle relative diffusion in isotropic turbulence. Phys. Fluids 6, 3416-3428 (1994)

26. Durbin, P.A.: A Stochastic-model of two-particle dispersion and concentration fluctuations in homogeneous turbulence. J. Fluid Mech. 100, 279-302 (1980) 
27. Bourgoin, M., Ouellette, N.T., Xu, H.T., Berg, J., Bodenschatz, E.: The role of pair dispersion in turbulent flow. Science 311, $835-838$ (2006)

28. Pumir, A., Shraiman, B.I., Chertkov, M.: Geometry of Lagrangian dispersion in turbulence. Phys. Rev. Lett. 85, 5324-5327 (2000)

29. Hackl, J.F., Yeung, P.K., Sawford, B.L.: Multi-particle and tetrad statistics in numerical simulations of turbulent relative dispersion. Phys. Fluids 23, 065103 (2011)

30. Devenish, B.J.: Geometrical properties of turbulent dispersion. Phys. Rev. Lett. 110, 064504 (2013)

31. Xu, H.T., Pumir, A., Bodenschatz, E.: The pirouette effect in turbulent flows. Nat. Phys. 7, 709-712 (2011)

32. Biferale, L., Boffetta, G., Celani, A., Devenish, B.J., Lanotte, A., Toschi, F.: Multiparticle dispersion in fully developed turbulence. Phys. Fluids 17, 111701 (2005)

33. Devenish, B.J., Thomson, D.J.: A Lagrangian stochastic model for tetrad dispersion. J. Turbul. 14, 107-120 (2013)

34. Pumir, A., Bodenschatz, E., Xu, H.T.: Tetrahedron deformation and alignment of perceived vorticity and strain in a turbulent flow. Phys. Fluids 25, 035101 (2013)

35. Bianchi, S., Biferale, L., Celani, A., Cencini, M.: On the evolution of particle-puffs in turbulence. Eur. J. Mech. B-Fluid 55, 324-329 (2016)

36. Yang, Y.: Identification, characterization and evolution of non-local quasi-Lagrangian structures in turbulence. Acta Mech. Sin. 32, 351-361 (2016)

37. Sagaut, P.: Large Eddy Simulation for Incompressible Flows. An Introduction. Springer, Berlin (1998)

38. Lesieur, M., Metais, O., Comte, P.: Large-Eddy Simulation of Turbulence. Cambridge University Press, Cambridge (2005)

39. Fang, L., Zhu, Y., Liu, Y.W., Lu, L.P.: Spectral non-equilibrium property in homogeneous isotropic turbulence and its implication in subgrid-scale modeling. Phys. Lett. A 379, 2331-2336 (2015)

40. He, G.W., Jin, G.D., Yang, Y.: Space-time correlations and dynamic coupling in turbulent flows. Annu. Rev. Fluid Mech. 49, 51-71 (2017)

41. Chollet, J.P., Lesieur, M.: Parameterization of small scales of three-dimensional isotropic turbulence utilizing spectral closure. J. Atmos. Sci. 38, 2747-2757 (1981)

42. Chollet, J.P.: Two-Point Closure used for a Sub-Grid Scale Model in Large Eddy Simulations. Turbulent Shear Flow 4, pp. 62-72. Springer, Berlin (1983)

43. Ray, B., Collins, L.R.: Preferential concentration and relative velocity statistics of inertial particles in Navier-Stokes turbulence with and without filtering. J. Fluid Mech. 680, 488-510 (2011)

44. Yi, C., Li, J., Liu, Z.H., Wang, L., Zheng, C.G.: Subgrid-scale fluid statistics along the inertial particle trajectory in isotropic turbulence. Chin. Phys. Lett. 29, 094701 (2012)

45. Armenio, V., Piomelli, U., Fiorotto, V.: Effect of the subgrid scales on particle motion. Phys. Fluids 11, 3030 (1999)

46. Fede, P., Simonin, O.: Numerical study of the subgrid fluid turbulence effects on the statistics of heavy colliding particles. Phys. Fluids 18, 045103 (2006)

47. Marchioli, C., Salvetti, M.V., Soldati, A.: Some issues concerning large-eddy simulation of inertial particle dispersion in turbulent bounded flows. Phys. Fluids 20, 040603 (2008)

48. Kuerten, J.G.M., Vreman, A.W.: Can turbophoresis be predicted by large-eddy simulation? Phys. Fluids 17, 011701 (2005)

49. Pozorski J., Luniewski M.: Analysis of SGS particle dispersion model in LES of channel flow. Quality and Reliability of LES 331-342 (2008)

50. Gobert, C., Manhart, M.: A priori and a posteriori analysis of models for large-eddy simulation of particle-laden flow. Phys. Fluid Dyn. 1004, 1-18 (2011)

51. Innocenti, A., Marchioli, C., Chibbaro, S.: Lagrangian filtered density function for LES-based stochastic modelling of turbulent dispersed flows. Phys. Fluids 28, 115106 (2016)

52. Yang, Y., He, G.W., Wang, L.P.: Effects of subgrid-scale modeling on Lagrangian statistics in large-eddy simulation. J. Turbul. 9, 1-24 (2008)

53. Chow, F.K., Moin, P.: A further study of numerical errors in large-eddy simulations. J. Comput. Phys. 184, 366-380 (2003)

54. Bianco, F., Chibbaro, S., Marchioli, C., Salvetti, M.V., Soldati, A.: Intrinsic filtering errors of Lagrangian particle tracking in LES flow fields. Phys. Fluids 24, 045103 (2012)

55. Chibbaro, S., Marchioli, C., Salvetti, M.V., Soldati, A.: Particle tracking in LES flow fields: conditional Lagrangian statistics of filtering error. J. Turbul. 15, 22-33 (2014)

56. Marchioli, C., Salvetti, M.V., Soldati, A.: Appraisal of energy recovering sub-grid scale models for large-eddy simulation of turbulent dispersed flows. Acta Mech. 201, 277-296 (2008)

57. Pitton, E., Marchioli, C., Lavezzo, V., Soldati, A., Toschi, F.: Anisotropy in pair dispersion of inertial particles in turbulent channel flow. Phys. Fluids 24, 073305 (2012)

58. Batchelor, G.K.: The application of the similarity theory of turbulence to atmospheric diffusion. Q. J. R. Meteorol. Soc. 76, $133-146(1950)$

59. He, G.W., Rubinstein, R., Wang, L.P.: Effects of subgrid-scale modeling on time correlations in large eddy simulation. Phys. Fluids 14, 2186-2193 (2002)

60. He, G.-W., Wang, M., Lele, S.K.: On the computation of space-time correlations by large-eddy simulation. Phys. Fluids 16, 3859-3867 (2004)

61. Jin, G.D., He, G.W.: A nonlinear model for the subgrid timescale experienced by heavy particles in large eddy simulation of isotropic turbulence with a stochastic differential equation. New J. Phys. 15, 035011 (2013)

62. Mazzitelli, I.M., Toschi, F., Lanotte, A.S.: An accurate and efficient Lagrangian sub-grid model. Phys. Fluids 26, 095101 (2014)

63. Stolz, S., Adams, N.A.: An approximate deconvolution procedure for large-eddy simulation. Phys. Fluids 11, 1699-1701 (1999)

64. Park, G.I., Urzay, J., Bassenne, M., Moin, P.: A dynamic subgrid-scale model based on differential flters for LES of particleladen turbulent flows. Report, Annu. Res. Briefs 2015, Cent. Turbul. Res., Stanford Univ., pp. 17-26 (2015) 
65. Michalek, W.R., Kuerten, J.G.M., Zeegers, J.C.H., Liew, R., Pozorski, J., Geurts, B.J.: A hybrid stochastic-deconvolution model for large-eddy simulation of particle-laden flow. Phys. Fluids 25, 123302 (2013)

66. Yao, H.D., He, G.W.: A kinematic subgrid scale model for large-eddy simulation of turbulence-generated sound. J. Turbul. 10, 1-14 (2009)

67. Ray, B., Collins, L.R.: A subgrid model for clustering of high-inertia particles in large-eddy simulations of turbulence. J. Turbul. 15, 366-385 (2014)

68. He, G.W., Zhang, J.B.: Elliptic model for space-time correlations in turbulent shear flows. Phys. Rev. E 73, 055303(R) (2006)

69. Zhao, X., He, G.-W.: Space-time correlations of fluctuating velocities in turbulent shear flows. Phys. Rev. E 79, 046316 (2009)

70. He, G.W., Jin, G.D., Zhao, X.: Scale-similarity model for Lagrangian velocity correlations in isotropic and stationary turbulence. Phys. Rev. E 80, 066313 (2009) 